\title{
Topology, chiral and Polyakov loop transitions at finite density in two-colour QCD
}

\section{B. Allés*}

INFN Sezione di Pisa, Pisa, Italy

E-mail: alles@df.unipi.it

\section{D’Elia}

Dipartimento di Fisica, Università di Genova and INFN, Genova, Italy

E-mail: delia@ge.infn.it

\section{P. Lombardo}

INFN, Laboratori Nazionali di Frascati, Frascati, Italy

E-mail: mariapaola.lombardodlnf.infn.it

\begin{abstract}
The behaviour of the topological susceptibility $\chi$ in QCD with two colours and 8 flavours of staggered quarks is studied at nonzero temperature on the lattice across the finite density transition. It is shown that the signal of $\chi$ drops abruptly at the (pseudo-)critical chemical potential $\mu_{c}$, much as it happens at the finite temperature and zero density transition. The Polyakov loop and the chiral condensate undergo their transitions at the same potential $\mu_{c}$. At a value $\mu_{s}$ of the chemical potential, which satisfies $\mu_{s}>\mu_{c}$, Pauli blocking supervenes and the theory becomes quenched.
\end{abstract}

XXIVth International Symposium on Lattice Field Theory

July 23-28, 2006

Tucson, Arizona, USA

\footnotetext{
*Speaker.
} 


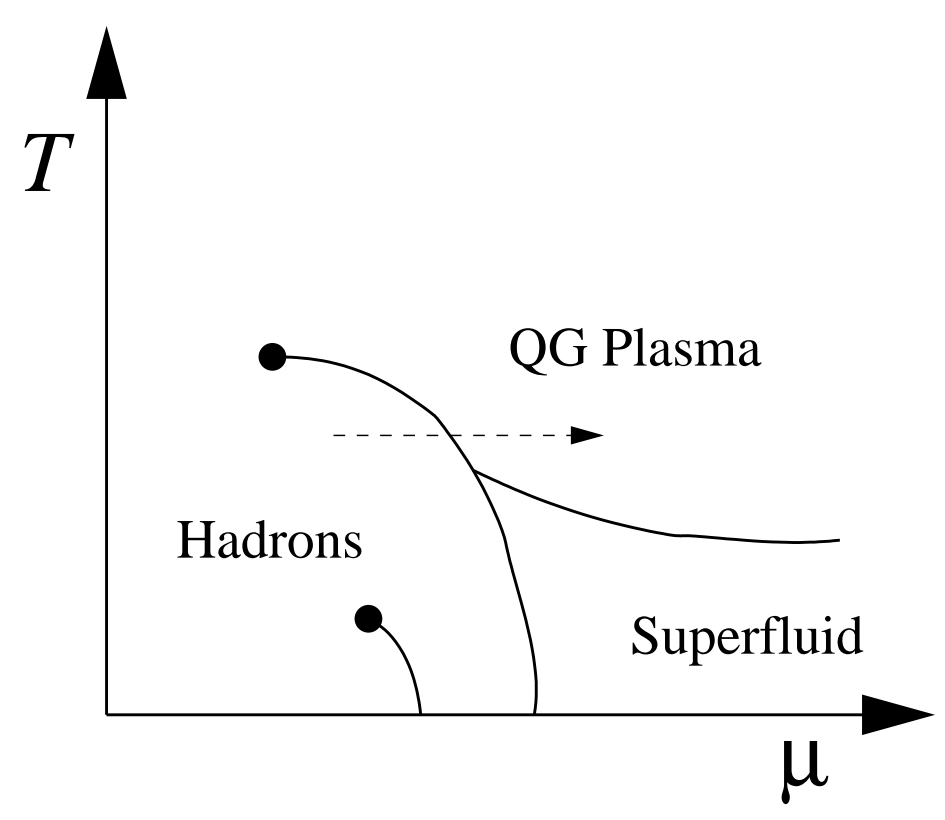

Figure 1: Sketch of the $T-\mu$ phase diagram in QCD with two colours. We have studied the transition crossed by the dashed arrow at finite temperature as the density is varied.

\section{Introduction}

In Fig. 1 the phase space of QCD in the temperature and quark chemical potential $\mu$ plane is shown schematically. Most of the features displayed in the Figure are theoretical predictions with marginal experimental verifi cation. We have studied the transition as the chemical potential is varied (dashed arrow in Fig. 1) at a fi xed temperature $T$ that is most likely above the region where a diquark condenses (see later).

We wanted to understand the fate of the topological susceptibility $\chi$ across the transition and to decide whether its possible change occurs at the same value of $\mu$ where the vector chiral symmetry is restored and the Polyakov loop signal rises [1].

It is known that at the fi nite temperature transition the signal of $\chi$ drops abruptly for gauge theories with two or three colours and several values of the flavour number [2, 3, 4]. The present is the fi rst work where a similar study is performed at the fi nite density transition (the full account can be found in Ref. [5]).

We have performed the study on the lattice by simulating the theory with two colours in order to avoid the sign problem that in the three colour theory makes the importance sampling method impracticable (for methods to overcome this problem see [6] and references therein). We expect that this modifi cation of true QCD has little impact on the results concerning gluon properties and in particular instanton physics. The main differences between two and three colour QCD regard aspects of the theory that we have not studied, like the hadron spectrum and the diquark condensed phase [7, 8]. 


\section{Simulation and observables}

We have simulated the model on a $14^{3} \times 6$ lattice at an inverse bare lattice gauge coupling $\beta=4 / g^{2}=1.5$ and quark mass $a m=0.07$ in units of lattice spacing $a$. The Wilson action was used for gauge fi elds and the staggered formulation for quarks. We introduced 8 flavours of staggered quarks.

The Hybrid Molecular Dynamics algorithm was chosen to update confi gurations. They were separated typically by 50-100 steps of the algorithm in order to well decorrelate the topology [9, 10, 11]. The behaviour of several observables was followed across the transition shown in Fig. 1: chiral condensate $\langle\bar{\psi} \psi\rangle$, Polyakov loop or Wilson line $P$, topological susceptibility $\chi$, baryonic density $\rho_{B}$ and average plaquette $\operatorname{Tr} \square / 2$.

The measurement of the topological susceptibility requires a careful treatment of composite operators. This quantity is defi ned as

$$
\chi \equiv \int \mathrm{d}^{4} x\langle\mathrm{~T}\{Q(x) Q(0)\}\rangle
$$

where $Q(x)$ is the topological charge density operator that appears in the r.h.s. of the axial singlet anomaly. $\chi$ is related to the $\eta^{\prime}$ mass through the Witten-Veneziano mechanism $[12,13]$ and to the chiral condensate in the massless limit [14].

Eq.(2.1) can be rewritten in a more compact form,

$$
\chi=\frac{\left\langle Q^{2}\right\rangle}{V}
$$

where the total topological charge $Q$ is defi ned as $Q \equiv \int \mathrm{d}^{4} x Q(x)$ and $V$ is the spacetime volume. This expression has only a formal meaning because it must be supplemented with a multiplicative renormalization for each power of the topological charge operator $Q[15,16]$ as well as a careful treatment of the contact divergences that appear when the two operators are evaluated at the same spacetime point [17].

A detailed description of the procedure that we have followed to extract $\chi$ can be found in [5]. In a nutshell, one has to defi ne a lattice regularization $Q_{L}(x)$ of the topological charge density operator. The corresponding total lattice topological charge is $Q_{L}=\sum_{x} Q_{L}(x)$ and the lattice topological susceptibility is $\chi_{L}=\left\langle Q_{L}^{2}\right\rangle / V$. This quantity is related to the physical susceptibility $\chi$ by the general expression $[15,16,17]$

$$
\chi_{L}=Z^{2} a^{4} \chi+M
$$

where $Z$ is a multiplicative renormalization of $Q_{L}, a$ is the lattice spacing and $M$ is an additive renormalization constant derived from the contact terms in Eq.(2.2). In presence of composite operators the usual renormalization program in a renormalizable theory is not enough to fully renormalize a Green's function. This is the origin of $Z$ (even in the case of the pure gauge theory where the topological charge operator has no anomalous dimensions). On the other hand part of the contact divergences that appear in the product of the two topological charge operators must be subtracted. This subtraction is defi ned as $\left.M \equiv \chi_{L}\right|_{Q=0}$, i.e. $M$ is the value of $\chi_{L}$ in the sector of zero topological charge. This condition guarantees the obvious requirement that $\chi$ vanishes in that sector. 


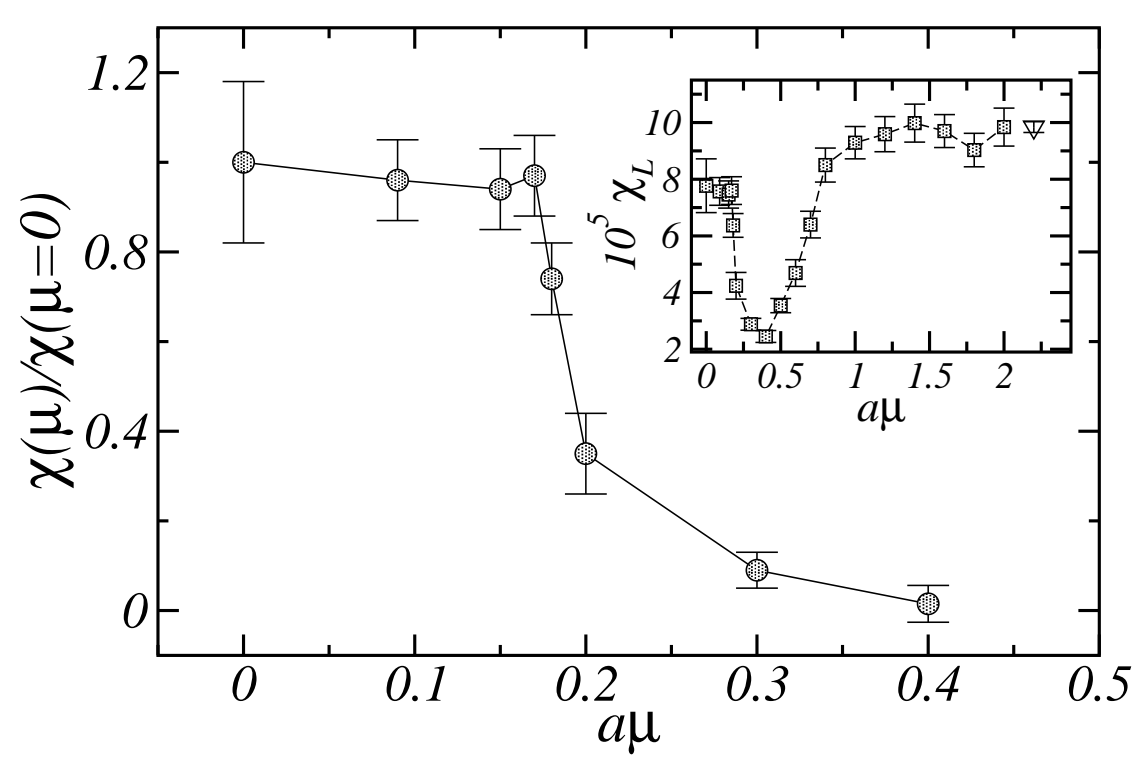

Figure 2: The ratio $\chi(\mu) / \chi(\mu=0)$ as a function of $a \mu$ showing the abrupt drop of the signal of the topological susceptibility at a (pseudo-) critical value of the chemical potential. In the inset the results for $\chi_{L}$ are displayed within a wide window of values of $\mu$.

We have obtained the topological susceptibility from Eq.(2.3) by using the "Pisa method" where the renormalization constants $Z$ and $M$ are explicitely calculated and then inserted in Eq.(2.3) in order to extract $\chi$. Since we shall present the results in the form of the ratio $\chi(\mu) / \chi(\mu=0)$ as a function of $\mu$ and since $Z$ is independent of infrared properties, hence of $\mu$, only the calculation of $M$ is required. This additive renormalization constant has been computed by using the nonperturbative method introduced in Refs. [18, 19,3]. It is enough to calculate it for one single value of the chemical potential because $M$ is independent of $\mu$ as well.

\section{Results}

In Fig. 2 we show the resulting behaviour of the topological susceptibility as a function of the chemical potential. A clear cut drop is seen at the position $a \mu_{c}=0.175(5)$. This sudden fall is an indication of the effective restoration of the axial singlet symmetry [20].

We do not show the analogous figures for the Polyakov loop and the chiral condensate behaviours (see Ref. [5]). Instead in Fig. 3 the derivatives of the three quantities (topological susceptibility, Polyakov loop and chiral condensate) are superimposed to give evidence of the coincidence of the three transitions: they all happen at the same (pseudo-)critical value $a \mu_{c}=0.175(5)$. Single points are the result of the discrete derivatives computed from the measured data while the lines are the derivatives of the interpolations made with a natural cubic spline [21] on each data set. The continuous line, corresponding to $(d \chi(\mu) / d \mu) / \chi(0)$, develops several maxima and minima. They are spurious effects on the spline interpolation that stem from the upward fluctuation of the latest point before the transition in Fig. 2. Since the theory deconfi nes at the same $\mu$ where $\langle\bar{\psi} \psi\rangle$ van- 


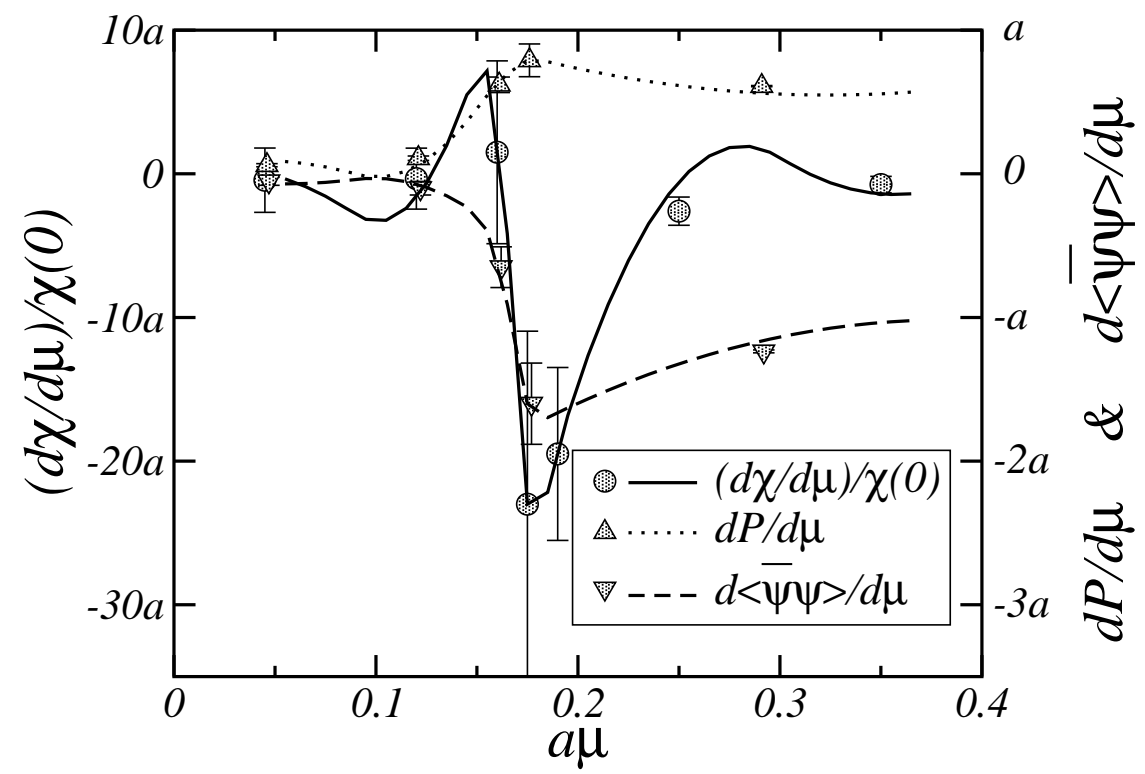

Figure 3: Derivatives with respect to the chemical potential $\mu$ of the normalized topological susceptibility (circles, continuous line and left vertical axis), Polyakov loop (up triangles, dotted line and right vertical axis) and chiral condensate (down triangles, dashed line and right vertical axis). The two vertical axes are expressed in units of the lattice spacing $a$. The three sets of data have been slightly shifted horizontally to avoid the overlapping of various symbols and error bars. Lines are the result of a spline interpolation.

ishes, the path in the $T-\mu$ diagram followed in our study likely lies above the superfluid phase (as indicated in Fig. 1) [22].

In order to give an estimate of the main results in physical units, we have done a second simulation of the model at zero chemical potential by varying the inverse bare lattice coupling $\beta$ on the same lattice volume and have measured the Polyakov loop and the chiral condensate. The data clearly reflected the existence of a critical $\beta_{c}=1.594(6)$. It was then assumed that the corresponding transition temperature $T_{c}$ lies in the range 100-200 MeV (this assumption was based on the following two facts: (i) the more fermions we include, the lower $T_{c}$ becomes and (ii) on the contrary $T_{c}$ increases with the mass of quarks which turn out to be rather heavy in our simulation). Hence a value for the lattice spacing at $\beta_{c}$ was obtained. This value was then run, by making use of the two-loop beta function, from $\beta_{c}$ to our working $\beta=1.5$ thus obtaining the lattice spacing $a(\beta=1.5)=0.64(4)\left(\begin{array}{c}+33 \\ -16\end{array}\right) \mathrm{fm}$. The errors are derived from the imprecision on $\beta_{c}$ and from the inaccuracy of the number assumed for $T_{c}$ respectively. We have not included an estimate of the error that stems from the use of the two-loop beta function to run $a$ from $\beta_{c}$ to $\beta=1.5$.

This result allowed us to assign physical units to the various parameters of our simulation: $\mu_{c}=54(2)(4)(18) \mathrm{MeV}$ (errors are respectively due to the estimate of $a \mu_{c}$, the imprecision on $\beta_{c}$ and the rough approximation of $T_{c}$ ) and $T=51(4)(17) \mathrm{MeV}$ (error on $\beta_{c}$ and on $T_{c}$ respectively).

Notice that the numerical value of $\mu_{c}$ is roughly compatible with half of the mass of the lightest baryon (which in the theory with two colours is made of two quarks and degenerated with the pion). However we take the above numbers with caution because of the large systematic errors 


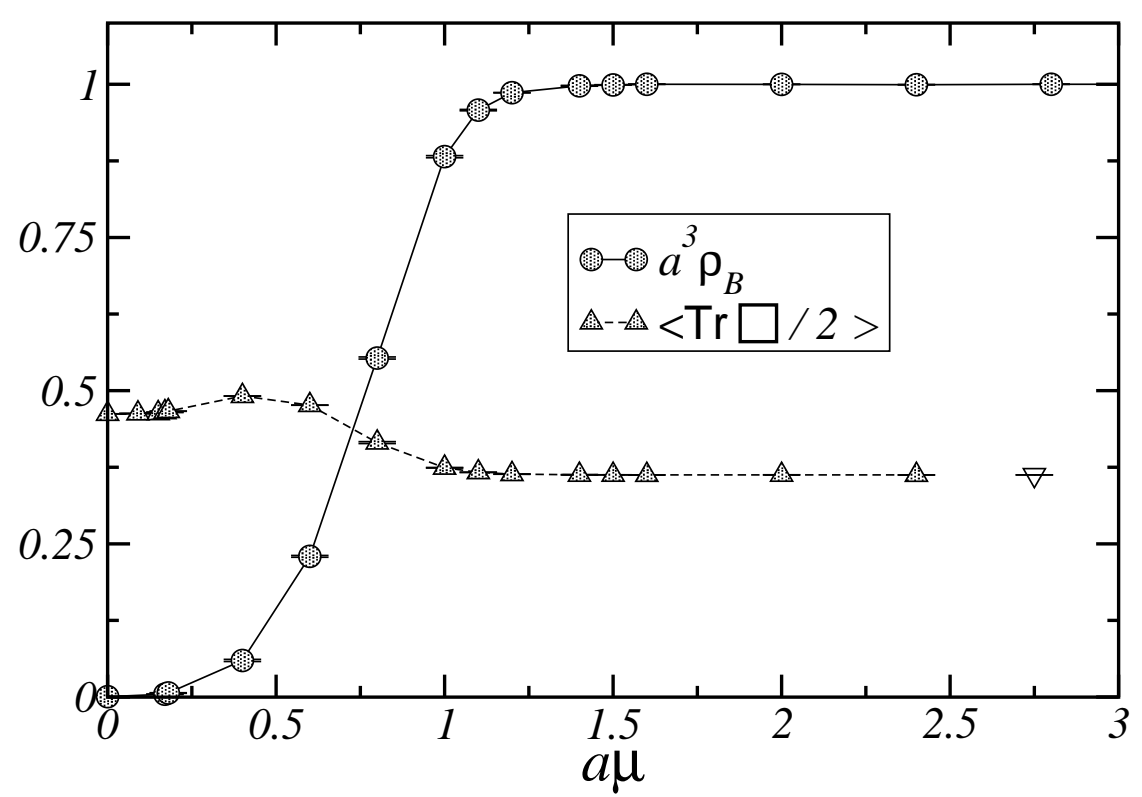

Figure 4: Baryon density per space site and average plaquette as a function of $a \mu$. The down triangle is the result of a separate simulation for the pure gauge theory.

derived from the small and coarse lattice volume, the (wrong) gauge group and the inexact updating algorithm.

\section{Pauli blocking}

By repeating the simulation at very high values of the chemical potential, in principle one could test the perturbative region of the phase diagram. However the advent of the so-called Pauli blocking makes it not viable. As $\mu$ increases, more and more fermions are placed in the lattice volume. Since it contains a countable number of sites, the Pauli principle imposes a maximum allowed number of fermions that can be placed on them. In our case the maximum allowed density is one baryon per site [23]. This maximum is attained for $a \mu_{s} \approx 1.2$. After that point, fermions are frozen and the theory becomes entirely quenched. We have verifi ed this statement by calculating the baryon density and the average plaquette. Their data are shown in Fig. 4. $\rho_{B}$ becomes 1 and stays constant for all $\mu>\mu_{s}$. Also the average plaquette stays constant after $\mu_{s}$ and this constant coincides with the value obtained from a separate Monte Carlo study where the pure gauge theory was simulated (represented by the down triangle in Fig. 4). The inset of Fig. 2 contains the data for $\chi_{L}$ which for $\mu>\mu_{s}$ reach a second plateau that coincides with the value obtained from an independent simulation using the pure gauge model (down triangle). We stress that Pauli blocking is a lattice effect with no counterpart in the continuum.

\section{Conclusions}

We have studied QCD with two colours and 8 flavours of staggered quarks on the lattice at 
fi nite temperature and chemical potential. We followed the behaviour of several observables across the transition run into by the dashed arrow depicted in Fig. 1. At the temperature $T$ which lies above the superfluid phase, the topological susceptibility $\chi$, the Polyakov loop and the chiral condensate undergo their transitions at the same value of $\mu$. Such a coincidence of transitions is the main result of our work. This value of $\mu$ is compatible with a critical chemical potential at zero temperature that equals half of the pion mass. This is the expected correct result, which however we take with caution because of the large quark mass used in the action. Rather this coincidence is likely due to a fortunate compensation of several systematic errors (approximate simulation algorithm, large number of quarks, wrong gauge group, estimate of $a(\beta=1.5)$, etc.).

\section{References}

[1] E. Shuryak, Comments Nucl. Part. Phys. 21 (1994) 235.

[2] B. Allés, M. D’Elia, A. Di Giacomo, Phys. Lett. B412 (1997) 119.

[3] B. Allés, M. D’Elia, A. Di Giacomo, Nucl. Phys. B494 (1997) 281, Erratum: ibid B679 (2004) 397.

[4] B. Allés, M. D’Elia, A. Di Giacomo, Phys. Lett. B483 (2000) 139.

[5] B. Allés, M. D’Elia, M. P. Lombardo, Nucl. Phys. B752 (2006) 124.

[6] E. Laermann, O. Philipsen, Ann. Rev. Nucl. Part. Sci. 53 (2003) 163.

[7] S. Hands, J. B. Kogut, M. P. Lombardo, S. E. Morrison, Nucl. Phys. B558 (1999) 327.

[8] J. B. Kogut, M. A. Stephanov, D. Toublan, J. J. M. Verbaarschot, A. Zhitnitsky, Nucl. Phys. B582 (2000) 477.

[9] B. Allés, G. Boyd, M. D’Elia, A. Di Giacomo, E. Vicari, Phys. Lett. B389 (1996) 107.

[10] B. Allés et al., Phys. Rev. D58 (1998) 071503.

[11] Y. Aoki et al., Phys. Rev. D72 (2005) 114505.

[12] E. Witten, Nucl. Phys. B156 (1979) 269.

[13] G. Veneziano, Nucl. Phys. B159 (1979) 213.

[14] F. C. Hansen, H. Leutwyler, Nucl. Phys. B350 (1991) 201.

[15] M. Campostrini, A. Di Giacomo, H. Panagopoulos, Phys. Lett. B212 (1988) 206.

[16] B. Allés, E. Vicari, Phys. Lett. B268 (1991) 241.

[17] M. Campostrini, A. Di Giacomo, H. Panagopoulos, E. Vicari, Nucl. Phys. B329 (1990) 683.

[18] A. Di Giacomo, E. Vicari, Phys. Lett. B275 (1992) 419.

[19] B. Allés, M. Campostrini, A. Di Giacomo, Y. Gündüç, E. Vicari, Phys. Rev. D48 (1993) 2284.

[20] R. D. Pisarski, F. Wilczek, Phys. Rev. D29 (1984) 338.

[21] W. H. Press, S. A. Teukolsky, W. T. Vetterling, B. P. Flannery, "Numerical Recipes in Fortran 77”, 2nd edition (1992), Cambridge University Press.

[22] J. I. Skullerud, S. Hands, S. Kim, hep-lat/0511001; D. Toublan, A. R. Zhitnitsky, hep-ph/0503256.

[23] H. Kluberg-Stern, A. Morel, O. Napoly, B. Petersson, Nucl. Phys. B220 (1983) 447. 